\title{
How to do physics by numbers
}

\section{Computers, widely used for solving intractable problems numerically, seem also to stimulate the imagination. Two recent examples confirm.}

THE simple truth that most problems in physics are not exactly soluble is widely appreciated. Generally speaking, there are two strategies for dealing with these awkward circumstances. One is to substitute for the real problem an idealization of it that can be tackled analytically, as when the motion of the Moon around the Earth is represented by one point mass travelling about another. The alternative is to fall back on mathematical approximations and the numerical methods that go with them, nicely exemplified by the complications of constructing accurate expressions for the motion of the real aspherical Moon around the aspherical (and inelastic) Earth in the presence of the Sun.

Now, with the arrival of computers, not merely is the position of the Moon easily and accurately calculable, but it is even possible to delegate to computer codes the tedious algebra entailed in working through the mathematical approximations. The consequence of such developments is not merely that numerical solutions have become more realistic, but that people's imaginations have been stimulated.

Quantum mechanics is an obvious field for computational innovation, if only because so few problems can be solved exactly. Those teaching the subject are hamstrung by the rarity of exact solutions of the Schrodinger equation. Similar difficulties crop up throughout the field, with the result that a few months ago A.H. Cooke thought it worth publishing a catalogue of the solutions of Dirac's equations (Proc. $R$. Soc. A383, 247-278; 1982).

In such circumstances, numerical methods of solution flourish, but even so it is something of a surprise that Carl $M$. Bender (from Washington University, St Louis) and David H. Sharp (from Los Alamos) should be able to announce the application of the method of finite elements, much loved by engineers, to the solution of equations describing operator fields (Phys. Rev. Lett. 50, 1535; 1983).

The method of finite elements, essentially what R. V. Southwell used to call the "relaxation" method, is simple enough when applied to the solution of a differential equation. For a first-order equation containing only first differential coefficients of some quantity, for example, it is sufficient to divide the range of the independent variable or variables into a large number of pieces of equal length and to represent the dependent variable whose solution is sought by a series of linear functions, one in each subdivision of the range. Requiring that the slope in each successive subdivision of the range should be that specified by the original differential equation then yields a string of numerical relationships between the values of the unknown quantity at the two ends of each subdivision of the range.

But what happens if the variables are not ordinary functions but, rather, operators in the sense of quantum mechanics? This is the starting point for the exercise that Bender and Sharp have undertaken. By way of illustration they take the example of a one-dimensional system, defined by a hamiltonian of the form $p^{2} / 2+V(q)$ where $p$ and $q$ are respectively the momentum and position variables and $V(q)$ is some potential function. Those bent on finding the stationary states of such a system know well enough how to proceed - convert the hamiltonian into a differential operator by the usual rules and look for wave functions corresponding to eigenstates. But what if time-dependence is the essence of the problem, so that both $p$ and $q$ are operators which are functions of the time $t$ ? The quantum mechanical analogues of Hamilton's classical equations are then $\mathrm{d} p(t) / \mathrm{d} t=f(q(t))$ and $\mathrm{d} q(t) / \mathrm{d} t=p(t)$ where the function $f$ is $-\mathrm{d} V / \mathrm{dq}$.

So, in practice, it is feasible to think of solving this pair of simultaneous first-order equations for $p$ and $q$ by the method of finite elements, chopping up the range of time into suitably small elements and starting with suitable initial values for the two operators. The snag is that such an interaction will yield nonsense unless the operators $p$ and $q$, conjugate momentum and position as they are, satisfy the commutation relations $q(t) p(t)-p(t) q(t)=i$ (in units such that $h / 2 \pi=1$ ) at all points bounding subdivisions of the time range. What Bender and Sharp have done is to show that the commutation relations are indeed preserved by the iteration process and then, by heroic algebra, that the corresponding conditions survive in the iterative time-evolution of a simple scalar field. The chief value of their method will be to sharpen physical intuition.

Much the same may be true of the technique described by Michael Creutz (from Brookhaven National Laboratory) for calculating the partition function of some physical system (Phys. Rev. Lett. 50, 1411; 1983). Since the time of Willard Gibbs, statistical mechanics has been based on the argument that the entropy of a system can be calculated from the number of configurations compatible with such external constraints as there may be, for example, its fixed energy.

The practice of statistical mechanics is in essence the calculation of the numbers of configurations compatible with this or that constraint, but with the advent of computers people have recognized that many intractable jobs can be done, at least numerically, by machine. One way of tackling such problems is by a version of the Monte Carlo method - set up a computer model of the microscopic components of a system, let the system evolve in the course of time but randomly, program the machine to recognize distinct configurations . . . and simply let it run. For then, by Poincaré's ergodic theorem, the system will eventually run through all possible configurations compatible with the constraints. Problems such as that of a lattice whose vertices carry entities which interact with their neighbours are conveniently dealt with, and have the added attraction that they may also yield solutions to problems in the gauge theory of particle fields (see Nature 299, 675; 1982).

Creutz's amusing innovation in this process is essentially a trick for simplifying the imposition of external constraints. $\mathrm{He}$ postulates (and builds into his programs) a microscopic "demon" that wanders from place to place, using a portable stock of energy to change the state of some microscopic element. The rules of the game are that the demon puts in his "sack" such energy as it manages to collect, but is not able to change the state of a microscopic component if the result would that his own stock of energy was exhausted.

One advantage of this arrangement is its simplicity. Programming demons is child's play Moreover, the mere existence of a demon carrying part of the energy of the system turns that into what Gibbs called a microcanonical ensemble - a system large enough for thermodynamic quantities such as temperature to be defined, but which is nevertheless part of some larger system. The result is that the average energy carried by the demon can be directly related to the temperature. Creutz's own interest in gauge theories leads him to offer illustrations of the working of his technique in the context of SU(2) theory, but he also points to the intriguing possibility that a demon could be programmed to conserve more interesting quantities.

John Maddox 